\title{
Understanding Small-Scale Structure Formation: Are We There Yet?
}

\author{
James E. Taylor* \\ Department of Physics and Astronomy, University of Waterloo \\ 200 University Avenue West, Waterloo, Ontario, N2L 3G1, Canada \\ E-mail: taylor@uwaterloo.ca
}

\begin{abstract}
After decades of dedicated experimental work, the identification of dark matter may soon be upon us. Within the coming year, the Large Hadron Collider (LHC) will reach electroweak-scale energies where it may be able to produce new particles directly, while direct and indirect detection experiments should soon have the sensitivity to search large regions of parameter space for dark matter candidates. To properly interpret any astrophysical signals we detect in these experiments requires an accurate model of the distribution of dark matter in and around the Milky Way. The theoretical problem of predicting this distribution down to the smallest scales is further from being resolved than is sometimes claimed. I discuss the difficulties inherent in making accurate predictions for direct and indirect detection signals using n-body techniques, and present some initial results obtained using an alternative modelling technique. I also point out some of the new cosmological tests to which we will have access when small-scale dark matter structure is finally detected.
\end{abstract}

Identification of dark matter 2008

August 18-22, 2008

Stockholm, Sweden

\footnotetext{
${ }^{*}$ Speaker.
} 


\section{Introduction}

The current evidence for dark matter comes from a diverse set of observations over a wide range of scales and redshifts, ranging from the fluctuation spectrum of the cosmic microwave background at $z=1100$ to the dynamics of local dwarf galaxies at $z=0$. If the missing component needed to explain these observations is indeed cold dark matter (CDM), it represents a major new addition to the Standard Model's inventory of particles and forces, and its identification is an important goal for experimental high-energy physics. While detecting dark matter or its annihilation products experimentally is extremely challenging, the rapid and concurrent progress in several branches of this field suggests that we may be on the verge of a major experimental discovery.

Perhaps it is fitting, then, to ask whether theory has kept pace. Theoretical models of structure formation should provide two specific predictions: first, how dark matter is distributed in and around the Solar System, that is on scales much smaller than our Galaxy, and second, to what extent the dark matter distribution in clumped on all scales throughout the Galaxy. These predictions will allow us to interpret the result of 'direct' (laboratory) and 'indirect' (observational) detection experiments respectively. They will be particularly important if the experiments see little or no signal, in which case models of the distribution will allow us to translate upper limits in detection into limits on particle properties, or if we succeed in determining the candidate particle's properties without detecting its spatial distribution, e.g. through measurements at the LHC. Furthermore, identifying dark matter is not the end of the story; if the small-scale distribution of CDM can be mapped it will provide a whole new window, inaccessible by other means, onto the physics of the early universe. Clearly the modellers need to be ready.

So how is the modelling of small-scale structure progressing? As experiments have improved, so too has our understanding of structure formation in the dark component. With the spectacular success of n-body simulations, in particular, we now have a good understanding of the formation of dark matter halos, their density profiles, concentration, shape, spin and other properties. We have been able to resolve dense substructure within halos for about 10 years, and may have reached the point where we can reliably extrapolate from these simulations down to the tiny scales relevant to direct and indirect detect detection experiments. But the extrapolation required is very large 10 orders of magnitude or more - and the results are sensitive to the details of the calculation, as demonstrated by the range of conclusions reached even in these proceedings [1, 2]. It is worth going over this problem very carefully, before we persuade ourselves that we have resolved it fully.

\section{Why is predicting the local dark matter distribution so hard?}

Suppose we consider the basic picture of small-scale structure formation for a specific CDM candidate, such as a supersymmetric weakly-interacting massive particle (WIMP); many aspects of the picture will then be generic to other candidates. As the early universe cools, these WIMPs drop first out of chemical (annihilation) equilibrium, and then out of kinematic equilibrium with other species. A combination of these processes and subsequent free-streaming sets the smallest scale on which WIMPs trace the primordial fluctuations from inflation; detailed calculations [3] indicate this minimum scale should range from $\sim 10^{-4}$ to $10^{-8} M_{\odot}$ or less. 
These fluctuations will then evolve and grow to the present day, and on scales above the baryonic Jeans mass (roughly $10^{6} M_{\odot}$ at high redshift) they will collect baryons and form visible stars grouped into small galaxies. Subsequent hierarchical merging between these small units should lead to the formation of large structures like the Milky Way by a redshift of $\sim 1-2$. At the present day, we expect the Milky Way to consist of a single large dark matter halo with a central region several hundred times the density of the background at the time of its assembly, full of smaller substructures that correspond to the nuclei of partially disrupted halos from earlier stages of hierarchical assembly. The challenge for modellers is to predict the abundance, spatial distribution, density profile, and degree of tidal disruption for these 'subhalos' as a function of position inside a typical Galactic halo.

Clearly numerical simulations, with a current limiting resolution of $\sim 10^{9}$ particles, can't model the entire range of scales required directly. Alternate approaches include simulating a very small region at high redshift to determine its initial substructure content [4], generating full simulations down to $z=0$ and studying their convergence as resolution increases [1,2], or using analytic or semi-analytic approximations to extend purely numerical models $[5,6]$.

The predictions required are particularly demanding. For direct detection we need to know the properties of the smallest subhalos (with masses $10^{-18}$ times that of the main halo or less) at the Solar radius, that is deep within the main halo in a region heavily influenced by baryons. For indirect detection, we need to know the innermost density profile of the densest subhalos, that is the subhalos that formed earliest and on the smallest scales. The halo mass function observed in simulations has a logarithmic slope $d \ln (n) / d \ln (m) \sim-2$, which means that different decades contribute almost equally to the mass fraction in substructure. The direct detection annihilation signal from a single subhalo goes as its concentration parameter $c^{2}-c^{3}$, and concentration $c$ in turn increases with increasing formation redshift or decreasing mass. Thus small, dense subhalos can contribute strongly to the annihilation signal. Yet these objects are the hardest to resolve in simulations, particularly at high redshift where the mean density is high and the fluctuations are small. Resolution effects made a noticeable difference to the predictions of a previous generation of n-body simulations [5]. It may be that the current generation of has finally overcome this problem, but it is important to test their predictions very carefully using other techniques.

\section{Some results from an alternative approach}

An alternative to direct simulation of halo substructure is to build a hybrid model, based partly on numerical integration and partly on analytic approximations. This technique is more cumbersome, but has the advantage of focussing computational power where it is needed, while glossing over large-scale patterns that are already well-established and of less direct interest. Thus, for instance, one can use analytic approximations and Monte-Carlo methods to specify how a halo accretes mass through mergers, and how its shape, density profile, and concentration parameter change. Individual subhalos can then be evolved in this smooth main halo to determine how their mass, density profile and orbital parameters evolve, using direct integration or analytic approximations. In previous work I have developed a method of this kind based on mass accretion histories from semi-analytic merger trees [6]. The resulting model has been shown to reproduce the broad 


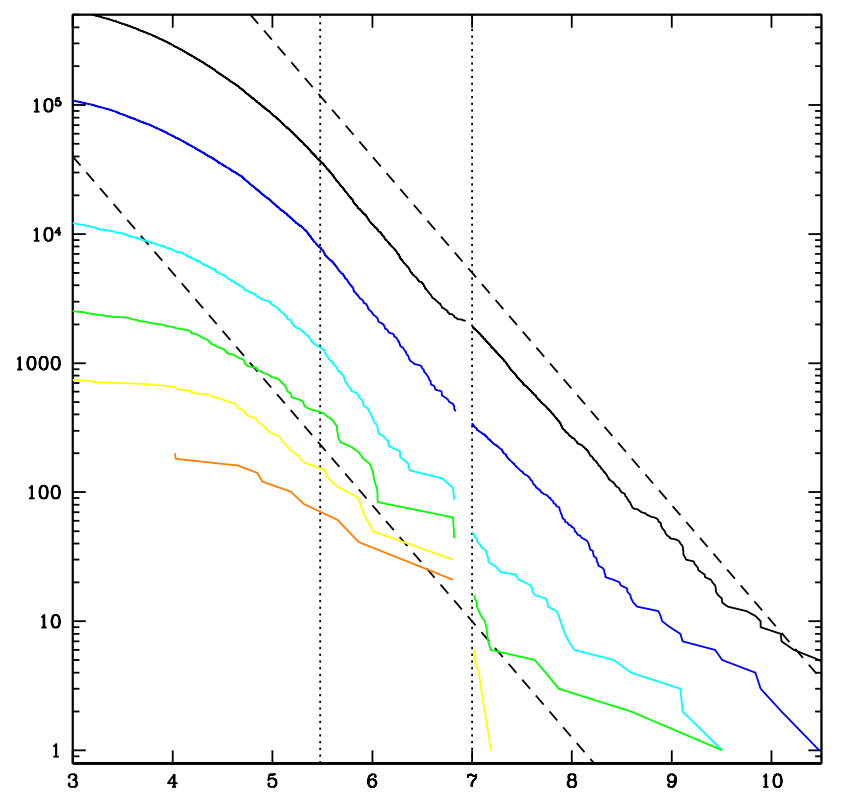

Figure 1: Cumulative mass functions for subhalos in a Milky-Way-sized halo within 1.2, 0.48, 0.24, 0.12, 0.06 and 0.03 of the virial radius from the centre (coloured lines, from top to bottom). Below $10^{7} M_{\odot}$ the merger tree has been sampled sparsely and the counts are rescaled to the full value expected for the whole tree, explaining the discontinuity in the mass functions at this point. Dashed lines indicate a slope of -1 .

patterns of a previous generation of $n$-body simulations, at a fraction of the computational expense [5].

Earlier versions of this model used a merger-tree code that only scaled well up to resolutions of $10^{5}-10^{6}$, i.e. subhalos with masses of $10^{7} M_{\odot}$. Recently I have modified the merger tree code so that it scales linearly with resolution, allowing it to generate very large trees. Figure 1 shows cumulative mass functions $N(<m)$ versus $\ln (m)$, for subhalos in a Milky-Way-sized halo extending down to a mass of $10^{3} M_{\odot}$. (Since the number of subhalos in the model becomes unnecessarily large at low masses, these mass functions were sampled sparsely below $10^{7} M_{\odot}$, that is only $5 \%$ of the branches in the merger tree were followed in detail, explaining the slight discontinuity in the figure at this mass. The results below this are scaled up by a factor of 20.) The different mass functions are for systems within 1.2, 0.48, 0.24, 0.12, 0.06 and 0.03 of the virial radius, from top to bottom. I obtain 200 subhalos within the 0.03 of the virial radius, that is roughly the Solar radius. These results are not particularly impressive compared to those the recent Aquarius [2] or Via Lactea [1] simulations, achieving similar mass resolution while making many simplifications and approximations. What is impressive is that this model took only a few hours to run on a single CPU, compared to the million CPU-hours required for the largest self-consistent simulations. Given this speed, one can imagine extending this method to much smaller scales than will ever be accessible to direct simulation. I am currently exploring on this possibility. 


\section{Cosmology in the the post-identification era}

The theoretical study of small-scale structure is currently in its infancy. If the CDM particle is identified and CDM structure can be mapped out observationally, however, a whole new range of applications may open up as a result. Current models of the early universe, for instance, rely on inferences about the shape and amplitude of the primordial power spectrum to constrain models of inflation. Only a limited amount of information can be derived from current observations, since they cover a limited range of scales. Measuring primordial power on very small scales would provide tremendous leverage for determinations of the tilt of the primordial spectrum, or in searches for any other features in the spectrum. The same is true for tests of scale-dependent non-gaussianity, which could produce extreme effects on small scales [7]. Thus if we can determine the abundance and clustering of dark subhalos on the smallest scales, we will learn something important about the earliest moments in the history of the universe.

\section{Summary}

Given the rapid progress on several experimental fronts, we may soon be in a position to detect dark matter directly in accelerators or lab experiments, or indirectly via its decay products using neutrino, gamma-ray, or antimatter observatories. It is not clear if the models of smallscale structure formation required to interpret these results are comparably mature. Predicting the distribution of dark matter to the level required by current experiments is extremely challenging, for a number of fundamental reasons. Thus, while recent numerical simulations have made great strides in modelling dark matter halos at very high resolution, it is still not clear whether they represent the final word in this field. When we do finally detect small-scale dark matter structure, it will open up many new and previously unforeseen tests of early-universe cosmology.

\section{References}

[1] M. Kuhlen, in these proceedings (Identification of dark matter 2008, 2008 )

[2] C. Frenk, in these proceedings (Identification of dark matter 2008, 2008)

[3] e.g. S. Profumo, K. Sigurdson, \& M. Kamionkowski, What Mass Are the Smallest Protohalos?, PRL 97 (2006) 031301 [astro-ph / 0603373 ].; T. Bringmann \& S. Hofmann, Thermal decoupling of WIMPs from first principles, JCAP 04 (2007) 016 [hep-ph / 0 612238].

[4] J. Diemand, B. Moore, \& J. Stadel, Earth-mass dark-matter haloes as the first structures in the early Universe, Nature 433 (2005) 389 [astro-ph / 0501589 ].

[5] J.E. Taylor \& A. Babul, The Evolution of Substructure in Galaxy, Group and Cluster Haloes III: Comparison with Simulations, MNRAS 364 (2005b) 535 [astro-ph / 0410049 ].

[6] J.E. Taylor \& A. Babul, The Evolution of Substructure in Galaxy, Group and Cluster Haloes II: Global Properties, MNRAS 364 (2005a) 515 [astro-ph / 041004 8]; J.E. Taylor \& A. Babul, The Evolution of Substructure in Galaxy, Group and Cluster Haloes I: Basic Dynamics, MNRAS 348 (2004) 811 [astro-ph/0301612];

[7] M. LoVerde, A. Miller, S. Shandera, \& L. Verde, Effects of Scale-Dependent Non-Gaussianity on Cosmological Structures, JCAP 04 (2008) 014 [ast ro-ph / 0711 . 4126]. 